\title{
Metabolic remodeling during murine early embryo development
}

Jing Zhao

Zhejiang University

Ke Yao

Tsinghua University

Hua Yu

Zhejiang University

Ling Zhang

Zhejiang University

Yuyan Xu

Zhejiang University

Zeping Hu ( $\nabla$ zeping_hu@tsinghua.edu.cn )

Tsinghua University

Jin Zhang ( $\nabla$ zhgene@zju.edu.cn )

Zhejiang University

Lang Chen

Zhejiang University

Zhen Sun

Zhejiang University

Yuqing Zhu

Zhejiang University

Chen Zhang

Yuli Qian

Shuyan Ji

Min Zhang

Jie Chen

Cristina Correia

Taylor Weiskittel

Da-Wei Lin

Sriram Chandrasekaran

Xudong Fu

Dan Zhang

Heng-Yu Fan

Wei Xie 
$\mathrm{Hu} \mathrm{Li}$

Zeping Hu

Jin Zhang

\section{Research Article}

Keywords: mouse embryo development, metabolism, epigenetics, L-2-HG, L2hgdh

Posted Date: August 2nd, 2021

DOI: https://doi.org/10.21203/rs.3.rs-623821/v1

License: (9) This work is licensed under a Creative Commons Attribution 4.0 International License. Read Full License 


\section{Abstract}

During early mammalian embryogenesis, dynamic changes in cell growth and proliferation are tightly linked to the underlying genetic and metabolic regulation. However, our understanding of metabolic reprogramming and its impact on epigenetic regulation in early embryo development remains elusive ${ }^{1}$. Here, we profiled metabolomes of embryos from the 2-cell and blastocyst stages, and their in vitro counterpart 2-cell like cells and ES cells, and reconstructed their metabolic landscape through the transition from totipotency to pluripotency. Our integrated metabolomics and transcriptomics analysis showed that 2-cell embryos favor methionine, polyamine and glutathione metabolism and stay in a more reductive state, whereas blastocyst embryos have higher mitochondrial TCA cycle metabolites and are in a more oxidative state. Moreover, we identify a reciprocal relationship between a-ketoglutarate $(\mathrm{a}-\mathrm{K} G)$ and the competitive inhibitor of a-KG-dependent dioxygenases L-2-hydroxyglutarate (2-HG) ${ }^{2}$, namely, higher L2-HG in the 2-cell embryos inherited from oocytes and 1-cell zygotes, and higher a-KG in the blastocyst. Supplementing 2-HG or knocking down $L 2 h g d h$, a gene encoding the 2-HG consuming enzyme L-2hydroxyglutarate dehydrogenase ${ }^{3}$ impeded erasure of global histone methylation markers ${ }^{4-6}$. Together, our data demonstrate dynamic and interconnected metabolic, transcriptional and epigenetic network remodeling during murine early embryo development.

\section{Introduction}

Metabolic reprogramming and its regulation during mammalian early embryo development are hardwired into the complex program of development ${ }^{7}$. Limitations in obtaining sufficient sample amounts and availability of reliable and scalable methods have hindered a deeper understanding of dynamic changes of metabolites, metabolic pathways and their upstream regulating factors during embryogenesis.

Classical analysis on whole embryos' physiology has focused on nutrition uptake, respiratory activity, and by-product secretion ${ }^{7}$. For instance, embryos utilize pyruvate earlier and later transition to glucose, and the knowledge led to the development of optimal media conditions currently used in in vitro fertilization clinical practice ${ }^{8-13}$. Moreover, blastocysts exhibit a respiratory burst during development ${ }^{14,15}$, but the underlying metabolic remodeling is still elusive. Recent omics analysis using low-input or single cell RNAseq and chromatin analysis has provided new insights into molecular regulatory circuitry governing early embryo development ${ }^{6,16-20}$. But metabolic genes are often labelled as "housekeeping" genes and metabolic alterations have been broadly neglected. Thus, a comprehensive picture of metabolic gene expression, their epigenetic status, and metabolite dynamics in early murine embryo development has yet to be uncovered.

Our limited knowledge of the metabolism molecular wiring in embryos is primarily built on studies with embryonic stem cells (ES cells), and their various pluripotent states representing different embryo stage counterparts $^{21-23}$, and studies with these cultured cells have provided key insights of their catabolic and anabolic metabolism ${ }^{24,25,26}$, and how metabolic networks are integrated into the well-established pluripotency networks such as Esrrb, Stat3, Lin28 and Zic3 in energy metabolism ${ }^{27-29}$, or c-Myc and 
$m$ Tor in anabolic metabolism ${ }^{30,31}$. However, there is a great need to confirm the physiological importance of these findings from cultured ES cells in the in vivo counterparts of embryo systems.

Epigenetic remodeling, especially histone methylation, is highly dynamic during pre-implantation embryogenesis ${ }^{4-6,19,20,32,33}$. How metabolites are involved in this process is largely unknown. Our findings for the first time provide insights into the role of reciprocal changes of a pair of competitive metabolites a-KG and 2-HG in the dynamic histone methylation erasure during early embryogenesis.

\section{Results}

\section{Metabolomics profiling revealed an elevated mitochondrial TCA cycle metabolic program during the 2-cell to blastocyst stage transition}

In order to understand the dynamic metabolic remodeling during pre-implantation embryo development, we employed mass spectrometry-based metabolomics to directly measure metabolite abundance in embryos. Due to difficulties in obtaining a large number of embryos and the limited detection range of current metabolomics technologies, we first need to optimize a targeted metabolomics approach to detecting metabolites using a small number of cells. We titrated a range of mouse ES cells from $2.5 \mathrm{k}$ to $80 \mathrm{k}$ cells (Extended Data Fig. 1a), or a range of zygotes from 15 to 240 embryos (Extended Data Fig. 1b), and we were able to achieve strong correlations between the number of cells/embryos and the mass spectrometry signal for most of the metabolites even at lower input ranges of the gradients (Extended Data Fig. 1c-e), indicating good quantification of those metabolite with this approach. Then we collected 100 2-cell stage embryos and 100 blastocyst stage embryos, which represent the totipotent state when zygotic genome activation takes place and the pluripotent state when ES cells can be derived ${ }^{34}$, and applied the targeted metabolomics method established above, each with three biological replicates (Fig. 1a). PCA analysis on targeted metabolite levels showed that 2-cell embryos were readily separated from blastocysts (Fig. 1b). Top differential metabolites include citrate, a-ketoglutarate (a-KG), succinate and glutamine and malate that are higher in the blastocyst stage, and 2-hydroxyglutarate(2-HG), Sadenosyl-methionine, GSSG, GSH, and spermidine that are higher in the 2-cell embryos (Fig. 1c and Extended Data Table 1). Notably, almost all the TCA cycle intermediate metabolites were more abundant in blastocyst embryos including citrate, succinate and a-KG $(p<0.05)$, but the abundance of the competitive inhibitor of a-KG-dependent dioxygenases ${ }^{2}$, the 2-HG, was higher in 2-cell embryos $(p<0.001$, Fig. 1d, e). Indeed, Metabolite Set Enrichment Analysis demonstrated "Citrate Acid Cycle" was among the top enriched metabolite sets in blastocyst embryos, whereas "Methionine Metabolism", "Spermidine and Spermine Biosynthesis" and "Nicotinate and Nicotinamide Metabolism" were among the top enriched metabolite sets in 2-cell embryos, respectively (Fig. 1f, g). Detailed analysis of the above metabolic pathways including the detected metabolites and the corresponding metabolic genes also revealed the dynamic metabolic signatures of the TCA cycle and purine metabolism pathways in the blastocyst embryos (Extended Data Fig. 2, 3), and one carbon metabolism as well as polyamine/glutathione/nicotinamide pathways related to the redox state in 2-cell embryos (Extended Data Fig. 4). For instance, higher level of the TCA cycle pathway metabolites in blastocysts is consistent 
with higher expression of the TCA cycle genes Aco1, Idh2, Sucla2, Sdha, Fh1 and Mdh2 (Figure S2), and higher level of GSH, GSSG, GSH/GSSH, spermidine and nicotinamide is associated with higher expression of the redox-related genes such as G6pdx (Extended Data Fig. 4).

To further analyze the metabolomics data in a systemic way, metabolites were mapped to a metabolic network based on our previous published method in genome-scale metabolic network modeling ${ }^{26}$ (Extended Data Fig. 5a), and differential genetic deletion sensitivity was predicted (Extended Data Fig. 5b). Consistently, 2-cell embryos are more sensitive to deletions of genes in redox-related "Glutathione metabolism", while blastocyst embryos are more sensitive to deletion of genes in "Oxidative phosphorylation" (Extended Data Table 2). In summary, our embryo metabolomics analysis revealed unique metabolic characteristics of 2-cell and blastocyst embryos.

It has been reported that the in vitro cultured ES cells have a spontaneously emerged 2-cell like cell (2CLC) population that resemble 2-cell embryos in their transcriptional signature ${ }^{21}$. To examine whether the metabolomes between embryos in vivo and their cultured counterparts in vitro are also similar, we applied the same metabolomics approach to ES cells and 2 CLCs. After transduced with a $2 \mathrm{C}$ reporter, 2C::tdTomato, the 2CLCs can be isolated from ES cell culture ${ }^{21}$. The same ES cell culture was also transduced with a Nanog::GFP reporter to indicate the pluripotent state ES cells (Extended Data Fig. 1f). We thus sorted 10,000 tdTomato positive 2CLCs or GFP positive ES cells each with three biological replicates for metabolomics analysis. In general, PCA analysis of common metabolites revealed that cultured cells clustered more closely to each other (Extended Data Fig. $5 \mathrm{c}$ ), indicating that the in vitro culturing condition has a strong impact on cellular metabolome. To lent further support, we performed metabolomics with another 2CLC system, a $2 \mathrm{C}$ gene Dux inducible ES cell line ${ }^{35}$, to enrich the tdTomato positive 2 CLCs. The ratio of 2 CLCs reached to $27.2 \%$ upon Dux induction (Extended Data Fig. $5 \mathrm{~d}, \mathrm{e}$ ), and the $2 \mathrm{C}$ genes were strongly activated (Extended Data Fig. $5 f$ ). We observed the similar patterns of the PCA analysis as the sorted 2CLC metabolomics from wild-type cells: globally, 2-cell and BC embryos clustered away from 2CLC and ES cells as they represented in vivo and in vitro samples (Extended Data Fig. $5 \mathrm{~g}$ ). Further comparing these 2CLCs and ES cells in the heatmap showed GSH, GSSG, GSH/GSSG, and spermidine are higher in 2CLC (Extended Data Fig. 5h-j), consistent with the analysis from embryos (Extended Data Fig. 1g and 4d,f,g). For better visualization, we overlapped metabolites higher in 2-cell embryos (compared to BC) and higher in 2CLC (compared to ES) (Fig. 1h,i), and they were specifically enriched in spermidine, methionine and glutathione metabolism, etc (Fig. 1j,k).Together, our embryo and cultured cell metabolomic analyses demonstrate that the embryo metabolome is distinguishable from that of their in vitro counterpart cells. Selective metabolic signatures show consistent trends, such as a more reduced metabolism in 2-cell embryos and 2CLCs, and a more oxidative state in blastocyst embryos and ES cells.

\section{Common and unique metabolic signatures revealed by integrated transcriptional and metabolic analysis}


RNA-seq and ChIP-seq analyses of early embryos have shed light on the dynamic genetic and epigenetic programs of these stages, including genes in metabolism. To further examine embryo metabolic features from a genetic regulation perspective, we re-analyzed publicly available bulk and scRNA-seq data in mouse embryos of different stages ${ }^{20,36}$, and identified "Energy Metabolism" and "Translation" as the two most drastically and consistently altered gene categories in early embryo development (Fig. 2a and Extended Data Fig. 6a). We then examined the 3000 metabolism-associated genes including metabolic enzymes or transporters ${ }^{37}$, and observed contrasting stage-specific gene expression patterns, and these metabolic genes were classified into 6 stage-specific clusters by k-means clustering for downstream analysis (Fig. 2b). Pathway enrichment analysis of the bulk RNA-seq data revealed that oxidative phosphorylation and citrate cycle (TCA cycle)-related genes were enriched in the blastocyst stage (equivalent to the ICM or Inner Cell Mass stage) gene cluster, consistent with the embryo metabolomics data, and phosphatidylinositol signaling-related genes were enriched in the MII-Oocyte and 2-cell stage clusters (Fig. 2c). Similarly, scRNA-seq data showed robust enrichment patterns for OxPhos and TCA cycle in the blastocyst stage, and phosphatidylinositol signaling in the zygote and 2-cell stage embryos (Extended Data Fig. 6b-c), together demonstrating that dynamic transcriptome with metabolic alterations are associated with early embryo development stages. When we examined the 2 CLC and ES cell transcriptomes ${ }^{21}$, we also found metabolic genes globally were not clustered with an embryo stage (Fig. 2b). However, GSEA analysis on selected gene sets such as"TCA cycle" and "Oxidative phosphorylation" showed higher expression in ES cells than 2CLCs (Extended Data Fig. 6d), consistent with the gene expression features in embryos.

To further validate the signature of "TCA cycle" and "OxPhos" (Extended Data Fig. 6e), we tested the ratio of NADH/NAD + during embryo development with a SoNar biosensor system by injecting in vitro transcribed SoNar mRNA into zygote embryos ${ }^{38}$. The ratio of NADH/NAD + from 2-cells to the blastocysts slightly increased, suggesting an increased mitochondrial TCA cycle and production of NADH (Extended Data Fig. 6f). This is consistent with the metabolomic analysis indicating that the mitochondrial TCA cycle oxidative metabolism is elevated in the blastocyst embryo (Fig. 1f). To validate a strong signature of "Translation" (Fig. 2a and Extended Data Fig. 6a) revealed by an increase of ribosome gene expression (Extended Data Fig. $6 \mathrm{~g}$ ), we determined nascent protein synthesis rate by OPpuromycin staining and found a marked increase from zygotes to morula/blastocyst embryos (Extended Data Fig. 6h), indicating an increased anabolic metabolism program as the embryos develop, which is critical to support the increased proliferation of embryonic cells. Interestingly, ribosome gene expression and translation activity peaked at 8-cell to morula stage, and slightly dropped at the blastocyst or ICM stage (Extended Data Fig. 6g), with a concomitant peak in OxPhos gene expression (Extended Data Fig. 6e), suggesting a unique state of slightly braked anabolic program, likely for embryos to be primed for the subsequent implantation process.

Materials from early embryos can be maternally inherited from oocytes. To distinguish the contribution of maternally-inherited versus zygotically transcribed metabolic signature, we also examined the chromatin status of the $\sim 3000$ metabolism-associated genes using previously published ATAC-seq ${ }^{17}$. ICM stage 
showed highest chromatin accessibility of ICM-specific metabolic genes (or Cluster 5 genes in Fig. 2b) determined by ATAC-seq peaks at transcription start sites (TSS), suggesting these genes are zygotically transcribed at the blastocyst stage (Extended Data Fig. 6i). In contrast, 2-cell stage-specific metabolic genes (or Cluster 2 genes in Fig. 2b) did not show significantly higher signals at TSS than ICM (Extended Data Fig. 6i), suggesting that their presence may not reflect active transcription, as most of genes at this stage are likely maternally inherited ${ }^{34}$. Importantly, our metabolomics analysis also identified 2-cell stagespecific metabolic pathways that were not revealed by gene expression analysis such as methionine metabolism (Fig. 1g, 2c). Comparing oocytes with 2C embryos showed that oocytes are also enriched in metabolites in this pathway such as methionine (Extended Data Fig. 1h), suggesting that certain metabolites from 2-cell stage embryos may be maternally inherited from oocytes. Together, our transcriptional analysis showed common and unique signatures compared with metabolomics analysis, demonstrating the importance of the integrated analysis to obtain a comprehensive understanding of embryo metabolism.

\section{The dynamic metabolic network is integrated into the developmental TF network in early embryogenesis}

The embryo development process is tightly governed by transcription regulation from developmental transcription factors (TF). We performed a TF binding motif enrichment analysis (Extended Data Fig. 6j) across embryo stage-specific clusters of metabolic genes (Fig. 2b). This analysis revealed that TFs like Esrrb, KIf, Myc and Nr5a2 are enriched in 8-cell/morula or ICM-specific metabolic genes' upstream regulators (Fig. 2d, Extended Data Fig. 6k). Next, co-expression profiles derived from RNA-seq and ChIPseq binding information were integrated to build a high confidence TF-metabolic gene regulatory network (Extended Data Fig. 6l) for each embryo stage-specific gene cluster (Fig. 2b). Increasingly complex regulatory networks were established from 2-cell/4-cell stages to 8-cell/morula and ICM stages (Extended Data Fig. 6I). Particularly, many mitochondrial TCA cycle and OxPhos-related genes such as Ndufa13, Ndufs2, Ndufc1, Ndufa1, Sdhc, Atp4a and Dlat were among the ICM TF targets. These regulatory networks are consistent with the transcriptome and metabolomics results implying the TCA cycle and OxPhos metabolism are highly activated during the blastocyst/ICM stage, but, more importantly, they demonstrate that the metabolic program is regulated by the classical developmental transcription factors. We also validated these findings with moues ES cell ChIP-seq data of key developmental transcription factors ${ }^{39}$, and found Esrrb, Myc and KIf genes were largely involved in the regulation of the TCA cycle and OxPhos genes with many shared target genes (Fig. 2e), in contrast to Sox2, Stat3 and Nanog which appeared to have very little or no influence in the TCA cycle and OxPhos metabolism gene regulation (Fig. 2e). Together, these results demonstrate that metabolic program are integrated into a specific set of upstream developmental TFs' regulatory network.

A reciprocal relationship between $\mathrm{a}-\mathrm{KG}$ and $2-\mathrm{HG}$ and a reduction of $\mathrm{L}-2-\mathrm{HG}$ facilitating global erasure of histone methylation during early embryogenesis 
Next, to understand whether metabolites are directly involved in gene regulation and embryo development, we chose a-KG and 2-HG which have established roles in epigenetic regulations and cell fate decision ${ }^{2,40,41}$. $a-K G$ is required for the enzyme activity of many dioxygenases such as histone demethylases, whereas D-2-HG is an oncometabolite produced by mutant IDH1/2, and it acts as an antagonist of a-KG to inhibit histone demethylation ${ }^{2}$. There are two enantiomers of 2-HG, D-2-HG and L-2$H G$, and the $L$ type $2-H G$ was recently found to be produced under certain physiological condition 42,43 . The detection of high 2-HG in early embryos when global epigenetic reprogramming take place made us to further explore the subtype and absolute concentration of this metabolite in MIl oocytes, 1-cell zygotes and 2-cell embryos. To our surprise, we found that L-2-HG, but not D-2-HG (Fig. 3a and Extended Data Fig. 7a), had the highest abundance in MIl oocytes, zygotes and 2-cell embryos, and it steadily decreased during the embryo development (Fig. 3b,c). The absolute concentration of L-2-HG we determined is in the millimole range, comparable to some cases of D-2-HG reported in IDH-mutation cancer cells or higher than L-2-HG reported in certain physiological conditions (Extended Data Fig. 7b,c) ${ }^{44-49}$. On the contrary, the absolute concentration of a-KG in blastocysts is more than 10-fold higher than 2-cell embryos (Fig. 3e). Even though a-KG is also slightly decreased from MIl oocytes to 2-cell embryos, the ratio of L-2$\mathrm{HG} / \mathrm{a}-\mathrm{KG}$ is markedly decreased throughout the early embryo development, from $\sim 6$-fold to less than 1fold (Fig. 3f,g). A decreasing L-2-HG concentration and decreasing L-2-HG/a-KG ratio after fertilization suggests that it might allow erasure of various histone methylation during this stage $\mathrm{e}^{4,6}$. To test this hypothesis, we treated embryos with a permeable L-2-HG octyl-L-2-HG50 during their in vitro development. Global erasure of $\mathrm{H} 3 \mathrm{~K} 4 \mathrm{me} 3$ and $\mathrm{H} 3 \mathrm{~K} 9 \mathrm{me} 3$ were indeed impeded or aberrant hyper-methylation was observed in the zygote to 4-cell stage embryos when supplementing ectopic L-2-HG from the zygote stage (Fig. 3h-k), with a non-toxic L-2-HG concentration that did not affect viability of 2-cell or 4-cell embryos (Extended Data Fig. 7d,e).

We also found delay of development process and embryo morphological abnormalities (Fig. 4a,b), such as the formed blastocyst embryos tended to collapse or not be able to hatch normally upon L-2-HG treatment (Fig. 4c,d), and the cavity area or averaged cell number per blastocyst decreased (Fig. 4e,f). Moreover, this effect at the blastocyst stage was more obvious when the treatment was restricted to earlier stages (Fig. 4g,h). RNA-seq analysis with L-2-HG-treated embryos that reached to early blastocyst stage showed a reduction in the TCA cycle and pluripotency genes (Fig. 4i). On the contrary, treatment with a permeable and embryo tolerable concentration of dimethyl-a-KG ${ }^{41}$ showed increased expression in the TCA cycle and pluripotent genes (Fig. 4j). Real-time PCR also validated increased Nanog expression upon a-KG treatment and decreased Nanog expression upon 2-HG treatment in blastocyst embryos (Fig. 4k,I). a-KG also rescued the L-2-HG effects on blastocyst formation rate, but could not fully rescue the morphological abnormalities (Fig. 4m,n), suggesting these two metabolites have certain opposing effects, but may also have other non-antagonizing effects ${ }^{51}$.

We next explored where the $\mathrm{L}-2-\mathrm{HG}$ is from and how it is cleared off. It has been reported that malate dehydrogenase $(\mathrm{MDH})$ or lactate dehydrogenase $(\mathrm{LDH})$ can produce $\mathrm{L}-2-\mathrm{HG}$ under certain physiological conditions $^{42,43,52}$, and L-2-hydroxyglutarate dehydrogenase $(\mathrm{L} 2 \mathrm{HGDH})$ can consume $\mathrm{L}-2-\mathrm{HG}^{3}$. We thus 
determined the expression levels of these enzymes with RNA-seq (Extended Data Fig. 8a), real-time PCR (Fig. 5a and Extended Data Fig. 8b) and western blotting analysis (Extended Data Fig. 8c). For both the mRNA and protein levels, $L d h b / L D H B$ is highly expressed in the oocytes, 1-cell and 2-cell embryos, with a gradual decrease during embryo development, and reaches to the lowest level at the blastocyst (ICM) stage. The other $L d h$ genes and $M d h 2$ do not show high expression of their mRNA at or before the 2-cell stage of the early embryo development. Simply knocking down a maternal factor $L d h b$ from zygote embryos may not interfere with L-2-HG abundance as it may be produced during oocyte development and inherited maternally from oocytes. Interestingly, mRNA level of $L 2 h g d h$ increased from 2-cell, and reaches to the highest level at 4-cell, and decreased afterwards (Fig. 5a). Its protein expression is also the highest at the 2-cell and 4-cell stages and decreases afterwards, suggesting it might have a role in consuming L2-HG during these stages. When knocking down L2hgdh by injecting siRNA in the zygote (Fig. 5b), we found an increase of L-2-HG in the 4-cell embryos (Fig. 5c). We also found H3K4me3 hyper-methylation in the 4-cell embryos, and in the presence of L-2-HG, this effect was further amplified (Fig. 5d-f), suggesting accumulation of L-2-HG without a consumption enzyme deteriorated the histone methylation erasure process. Together, these data demonstrate that a reduction of L-2-HG after fertilization and during preimplantation embryo development is required for the global erasure of histone methylation, and that accumulation of L-2-HG impedes this epigenetic remodeling process and influences embryo development.

\section{Discussion}

Mammalian embryogenesis is a complex process with coordinated regulation at multiple molecular levels. Using pooled mouse early embryos and optimized mass spectrometry based ultra-sensitive targeted metabolomics for a small number of cells we previously developed ${ }^{53}$, here we provided a comprehensive and quantitative metabolomics profiling of key stages in murine early embryo development. By integrating this data with transcriptome, chromatin states, and single cell profiling data on 3000 metabolism-associated genes, and by performing metabolic network analysis, we delineated the first metabolic landscape of murine pre-implantation embryo development. Notably, snapshots of metabolomics cannot directly indicate metabolic pathway activity, and isotope tracing analyses on embryos merit further investigations.

Our comprehensive metabolomics and genomics analysis unraveled a redox state transition during preimplantation embryogenesis, which is consistent with previous functional analysis on redox or respiration ${ }^{14,54,55}$. Transition from a reduced state to an oxidative state is consistent with the high L-2-HG to high a-KG transition. We found this decrease of L-2-HG is required for histone erasure during early embryogenesis, establishing a role of metabolic regulation on embryo epigenome remodeling. Interestingly, the phenotypes of H3K4 hypermethylation and embryo collapse defects revealed by L-2-HG supplementation is reminiscent of embryos with $\mathrm{H} 3 \mathrm{~K} 4 \mathrm{me} 3$ demethylase genes $K d m 5 a / b$ knocked down, suggesting these histone modifications may play a role in the L-2-HG effect ${ }^{6}$. However, the effects of decreased L-2-HG in facilitating demethylation might be broader than one class of histone demethylases. 
For instance, we also observed increased $\mathrm{H} 3 \mathrm{~K} 9 \mathrm{me} 3$ and DNA methylation upon L-2-HG supplementation, and because the global active demethylation of $\mathrm{H} 3 \mathrm{~K} 4 \mathrm{me} 3$ at the early embryogenesis is well characterized ${ }^{6,19,56}$, we focused on H3K4me3 here. How L-2-HG's impact on various methylation marks collectively influences embryo development, and how the downstream specificity on different modifications and genes merit further investigation. Importantly, metabolism in early embryos have also been linked to histone acetylation and zygotic genome activation, implicating the broad range of interaction between metabolic and epigenetic regulation during early embryo development ${ }^{57}$.

The 2-cell and blastocyst metabolic signatures also indicate that embryos transition from a quiescent state to metabolically active state with high anabolic metabolism and mitochondrial TCA cycle to provide intermediate metabolites ${ }^{58}$. The OxPhos and TCA cycle peak at blastocyst embryo stage, which also produce high level of $\mathrm{a}-\mathrm{KG}$ that can in turn facilitates pluripotent gene expression and developmental process ${ }^{41}$. Along with the activation of the TCA and OxPhos metabolism genes, many pluripotency/developmental transcriptional factors are induced. In contrast to Nanog and Sox2, transcription factors such as Esrrb, c-Myc and Klf family members share many binding targets in the TCA and OxPhos genes, and they might coordinately contribute to activation of these genes. This neglected metabolic regulation adds to the developmental TFs' well-established roles in regulating pluripotency networks ${ }^{59,60}$, and thus these TFs can be defined as a new set of developmental regulators with dual roles in establishing both pluripotency and metabolic networks.

\section{Declarations}

\section{ACKNOWLEDGMENTS}

We thank Hengyu Fan, Jianzhong Sheng, Dan Ye, Yan Ni, Xu Huang, Chandel Navdeep, Andrew Intlekofer for helpful discussion and sharing of facilities. J.Z. is supported by the National Key Research and Development Program of China (2018YFA0107100, 2018YFA0107103, 2018YFC1005002), the National Natural Science Foundation projects of China $(31871453,91857116)$ and the Zhejiang Natural Science Foundation projects of China (LR19C120001). Z.H. is supported by the National Key Research and Development Program of China (2019YFA0802100, 2019YFA0802102). Author contributions: J.Z. and Z.H. designed and supervised the study. J.Z., K.Y., Y.X., L.C., Z.S., Y.Q., Z. J. and Y.Z. performed the experiments. K.Y. J.Z. and Z.H performed the metabolomics analysis. H.Y., C.Z., C.C, W.T., H.L., W.X., and D.Z. performed the bioinformatics analysis and contributed to writing and discussing the manuscript.

Animal procedures were carried out according to the Ethical Guidelines of the Zhejiang University Animal Care and Use Committee. The authors declare no competing interests.

\section{References}

1. Wu, D. et al. Glucose-regulated phosphorylation of TET2 by AMPK reveals a pathway linking diabetes to cancer. Nature 559, 637-641, doi:10.1038/s41586-018-0350-5 (2018). 
2. Xu, W. et al. Oncometabolite 2-hydroxyglutarate is a competitive inhibitor of alpha-ketoglutaratedependent dioxygenases. Cancer Cell 19, 17-30, doi:10.1016/j.ccr.2010.12.014 (2011).

3. Ye, D., Guan, K. L. \& Xiong, Y. Metabolism, Activity, and Targeting of D- and L-2-Hydroxyglutarates. Trends Cancer 4, 151-165, doi:10.1016/j.trecan.2017.12.005 (2018).

4. Xu, R., Li, C., Liu, X. \& Gao, S. Insights into epigenetic patterns in mammalian early embryos. Protein Cell, doi:10.1007/s13238-020-00757-z (2020).

5. Xia, W. \& Xie, W. Rebooting the Epigenomes during Mammalian Early Embryogenesis. Stem Cell Reports, doi:10.1016/j.stemcr.2020.09.005 (2020).

6. Dahl, J. A. et al. Broad histone H3K4me3 domains in mouse oocytes modulate maternal-to-zygotic transition. Nature 537, 548-552, doi:10.1038/nature19360 (2016).

7. Zhang, J. et al. Metabolism in Pluripotent Stem Cells and Early Mammalian Development. Cell Metab 27, 332-338, doi:10.1016/j.cmet.2018.01.008 (2018).

8. Chronopoulou, E. \& Harper, J. C. IVF culture media: past, present and future. Hum Reprod Update 21, 39-55, doi:10.1093/humupd/dmu040 (2015).

9. Conaghan, J., Handyside, A. H., Winston, R. M. \& Leese, H. J. Effects of pyruvate and glucose on the development of human preimplantation embryos in vitro. J Reprod Fertil 99, 87-95, doi:10.1530/jrf.0.0990087 (1993).

10. Brinster, R. L. Studies on the Development of Mouse Embryos in Vitro. li. The Effect of Energy Source. J Exp Zool 158, 59-68, doi:10.1002/jez.1401580106 (1965).

11. Brown, J. J. \& Whittingham, D. G. The roles of pyruvate, lactate and glucose during preimplantation development of embryos from F1 hybrid mice in vitro. Development 112, 99-105 (1991).

12. Gardner, D. K. Changes in requirements and utilization of nutrients during mammalian preimplantation embryo development and their significance in embryo culture. Theriogenology 49, 83-102, doi:10.1016/s0093-691x(97)00404-4 (1998).

13. Gardner, D. K. \& Lane, M. Culture and selection of viable blastocysts: a feasible proposition for human IVF? Hum Reprod Update 3, 367-382, doi:10.1093/humupd/3.4.367 (1997).

14. Houghton, F. D., Thompson, J. G., Kennedy, C. J. \& Leese, H. J. Oxygen consumption and energy metabolism of the early mouse embryo. Mol Reprod Dev 44, 476-485, doi:10.1002/(SICl)10982795(199608)44:4<476::AID-MRD7>3.0.C0;2-I (1996).

15. Leese, H. J. Metabolism of the preimplantation embryo: 40 years on. Reproduction 143, 417-427, doi:10.1530/REP-11-0484 (2012).

16. Xue, Z. et al. Genetic programs in human and mouse early embryos revealed by single-cell RNA sequencing. Nature 500, 593-597, doi:10.1038/nature12364 (2013).

17. Wu, J. et al. The landscape of accessible chromatin in mammalian preimplantation embryos. Nature 534, 652-657, doi:10.1038/nature18606 (2016).

18. Lu, F. et al. Establishing Chromatin Regulatory Landscape during Mouse Preimplantation Development. Cell 165, 1375-1388, doi:10.1016/j.cell.2016.05.050 (2016). 
19. Zhang, B. et al. Allelic reprogramming of the histone modification H3K4me3 in early mammalian development. Nature 537, 553-557, doi:10.1038/nature19361 (2016).

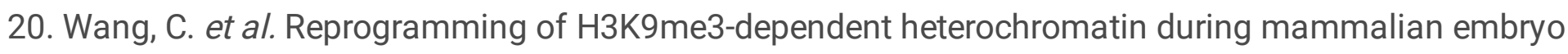
development. Nat Cell Bio/ 20, 620-631, doi:10.1038/s41556-018-0093-4 (2018).

21. Macfarlan, T. S. et al. Embryonic stem cell potency fluctuates with endogenous retrovirus activity. Nature 487, 57-63, doi:10.1038/nature11244 (2012).

22. Nichols, J. \& Smith, A. Naive and primed pluripotent states. Cell Stem Cell 4, 487-492, doi:10.1016/j.stem.2009.05.015 (2009).

23. Smith, A. Formative pluripotency: the executive phase in a developmental continuum. Development 144, 365-373, doi:10.1242/dev.142679 (2017).

24. Teslaa, T. \& Teitell, M. A. Pluripotent stem cell energy metabolism: an update. The EMBO journa/34, 138-153, doi:10.15252/embj.201490446 (2015).

25. Zhou, W. et al. HIF1a induced switch from bivalent to exclusively glycolytic metabolism during ESCto-EpiSC/hESC transition. The EMBO journa/31, 2103-2116, doi:10.1038/emboj.2012.71 (2012).

26. Chandrasekaran, S. et al. Comprehensive Mapping of Pluripotent Stem Cell Metabolism Using Dynamic Genome-Scale Network Modeling. Cell Reports 21, 2965-2977, doi:10.1016/j.celrep.2017.07.048 (2017).

27. Sone, M. et al. Hybrid Cellular Metabolism Coordinated by Zic3 and Esrrb Synergistically Enhances Induction of Naive Pluripotency. Cell Metabolism 25, 1103-1117.e1106, doi:10.1016/j.cmet.2017.04.017 (2017).

28. Carbognin, E., Betto, R. M., Soriano, M. E., Smith, A. G. \& Martello, G. Stat3 promotes mitochondrial transcription and oxidative respiration during maintenance and induction of naive pluripotency. The EMBO journa/ 35, 618-634, doi:10.15252/embj.201592629 (2016).

29. Zhang, J. et al. LIN28 Regulates Stem Cell Metabolism and Conversion to Primed Pluripotency Cell Stem Cell Article LIN28 Regulates Stem Cell Metabolism and Conversion to Primed Pluripotency. Cell Stem Cel/ 19, 66-80, doi:10.1016/j.stem.2016.05.009 (2016).

30. Scognamiglio, R. et al. Myc Depletion Induces a Pluripotent Dormant State Mimicking Diapause. Cell 164, 668-680, doi:10.1016/j.cell.2015.12.033 (2016).

31. Bulut-Karslioglu, A. et al. Inhibition of mTOR induces a paused pluripotent state. Nature 540, 119123, doi:10.1038/nature20578 (2016).

32. Eckersley-Maslin, M. A., Alda-Catalinas, C. \& Reik, W. Dynamics of the epigenetic landscape during the maternal-to-zygotic transition. Nat Rev Mol Cell Biol 19, 436-450, doi:10.1038/s41580-018-0008z (2018).

33. Burton, A. et al. Heterochromatin establishment during early mammalian development is regulated by pericentromeric RNA and characterized by non-repressive H3K9me3. Nat Cell Bio/ 22, 767-778, doi:10.1038/s41556-020-0536-6 (2020). 
34. Jukam, D., Shariati, S. A. M. \& Skotheim, J. M. Zygotic Genome Activation in Vertebrates. Dev Cell 42, 316-332, doi:10.1016/j.devcel.2017.07.026 (2017).

35. Fu, X., Djekidel, M. N. \& Zhang, Y. A transcriptional roadmap for 2C-like-to-pluripotent state transition. Science advances 6, eaay5181, doi:10.1126/sciadv.aay5181 (2020).

36. Deng, Q., Ramskold, D., Reinius, B. \& Sandberg, R. Single-cell RNA-seq reveals dynamic, random monoallelic gene expression in mammalian cells. Science 343, 193-196, doi:10.1126/science.1245316 (2014).

37. Birsoy, K. et al. An Essential Role of the Mitochondrial Electron Transport Chain in Cell Proliferation Is to Enable Aspartate Synthesis. Cell 162, 540-551, doi:10.1016/j.cell.2015.07.016 (2015).

38. Zhao, Y. et al. In vivo monitoring of cellular energy metabolism using SoNar, a highly responsive sensor for NAD(+)/NADH redox state. Nat Protoc 11, 1345-1359, doi:10.1038/nprot.2016.074 (2016).

39. Chen, $X$. et al. Integration of external signaling pathways with the core transcriptional network in embryonic stem cells. Cell 133, 1106-1117, doi:10.1016/j.cell.2008.04.043 (2008).

40. Lu, C. et al. IDH mutation impairs histone demethylation and results in a block to cell differentiation. Nature 483, 474-478, doi:10.1038/nature10860 (2012).

41. Carey, B. W., Finley, L. W., Cross, J. R., Allis, C. D. \& Thompson, C. B. Intracellular alpha-ketoglutarate maintains the pluripotency of embryonic stem cells. Nature $518,413-416$, doi:10.1038/nature13981 (2015).

42. Intlekofer, A. M. et al. Hypoxia Induces Production of L-2-Hydroxyglutarate. Cell Metab 22, 304-311, doi:10.1016/j.cmet.2015.06.023 (2015).

43. Intlekofer, A. M. et al. L-2-Hydroxyglutarate production arises from noncanonical enzyme function at acidic pH. Nat Chem Biol 13, 494-500, doi:10.1038/nchembio.2307 (2017).

44. Steinert, E. M., Vasan, K. \& Chandel, N. S. Mitochondrial Metabolism Regulation of T Cell-Mediated Immunity. Annual review of immunology 39, 395-416, doi:10.1146/annurev-immunol-101819082015 (2021).

45. Shim, E. H. et al. L-2-Hydroxyglutarate: an epigenetic modifier and putative oncometabolite in renal cancer. Cancer Discov 4, 1290-1298, doi:10.1158/2159-8290.CD-13-0696 (2014).

46. Amankulor, N. M. et al. Mutant IDH1 regulates the tumor-associated immune system in gliomas. Genes Dev 31, 774-786, doi:10.1101/gad.294991.116 (2017).

47. Terunuma, A. et al. MYC-driven accumulation of 2-hydroxyglutarate is associated with breast cancer prognosis. J Clin Invest 124, 398-412, doi:10.1172/jci71180 (2014).

48. Gross, S. et al. Cancer-associated metabolite 2-hydroxyglutarate accumulates in acute myelogenous leukemia with isocitrate dehydrogenase 1 and 2 mutations. The Journal of experimental medicine 207, 339-344, doi:10.1084/jem.20092506 (2010).

49. Bunse, L. et al. Suppression of antitumor T cell immunity by the oncometabolite (R)-2hydroxyglutarate. Nat Med 24, 1192-1203, doi:10.1038/s41591-018-0095-6 (2018). 
50. Tyrakis, P. A. et al. S-2-hydroxyglutarate regulates CD8(+) T-lymphocyte fate. Nature 540, 236-241, doi:10.1038/nature20165 (2016).

51. Fu, X. et al. 2-Hydroxyglutarate Inhibits ATP Synthase and mTOR Signaling. Cell Metab 22, 508-515, doi:10.1016/j.cmet.2015.06.009 (2015).

52. Oldham, W. M., Clish, C. B., Yang, Y. \& Loscalzo, J. Hypoxia-Mediated Increases in L-2hydroxyglutarate Coordinate the Metabolic Response to Reductive Stress. Cell Metab 22, 291-303, doi:10.1016/j.cmet.2015.06.021 (2015).

53. Agathocleous, M. et al. Ascorbate regulates haematopoietic stem cell function and leukaemogenesis. Nature 549, 476-481, doi:10.1038/nature23876 (2017).

54. Nasr-Esfahani, M. H. \& Johnson, M. H. Quantitative analysis of cellular glutathione in early preimplantation mouse embryos developing in vivo and in vitro. Human reproduction (Oxford, England) 7, 1281-1290, doi:10.1093/oxfordjournals.humrep.a137843 (1992).

55. Dumollard, R., Ward, Z., Carroll, J. \& Duchen, M. R. Regulation of redox metabolism in the mouse oocyte and embryo. Development 134, 455-465, doi:10.1242/dev.02744 (2007).

56. Liu, X. et al. Distinct features of H3K4me3 and H3K27me3 chromatin domains in pre-implantation embryos. Nature 537, 558-562, doi:10.1038/nature19362 (2016).

57. Nagaraj, R. et al. Nuclear Localization of Mitochondrial TCA Cycle Enzymes as a Critical Step in Mammalian Zygotic Genome Activation. Cell 168, 210-223 e211, doi:10.1016/j.cell.2016.12.026 (2017).

58. Vander Heiden, M. G., Cantley, L. C. \& Thompson, C. B. Understanding the Warburg effect: the metabolic requirements of cell proliferation. Science 324, 1029-1033, doi:10.1126/science.1160809 (2009).

59. Orkin, S. H. et al. The transcriptional network controlling pluripotency in ES cells. Cold Spring Harb Symp Quant Biol 73, 195-202, doi:10.1101/sqb.2008.72.001 (2008).

60. Ng, H. H. \& Surani, M. A. The transcriptional and signalling networks of pluripotency. Nat Cell Bio/ 13, 490-496, doi:10.1038/ncb0511-490 (2011).

\section{Figures}


Figure 1

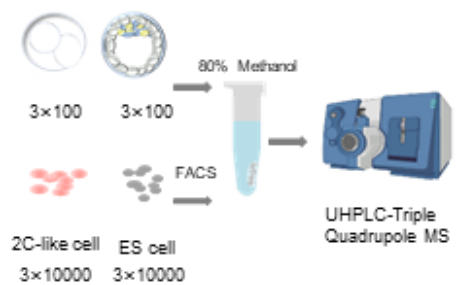

d

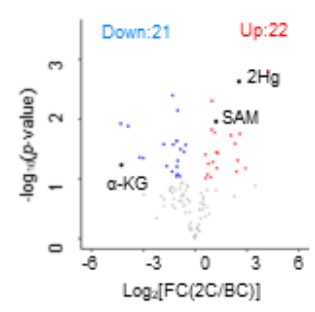

b

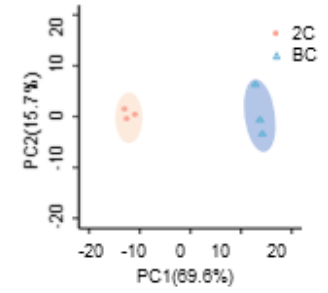

C
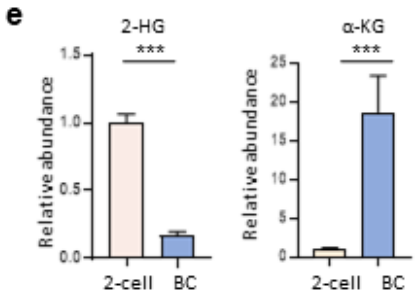

f
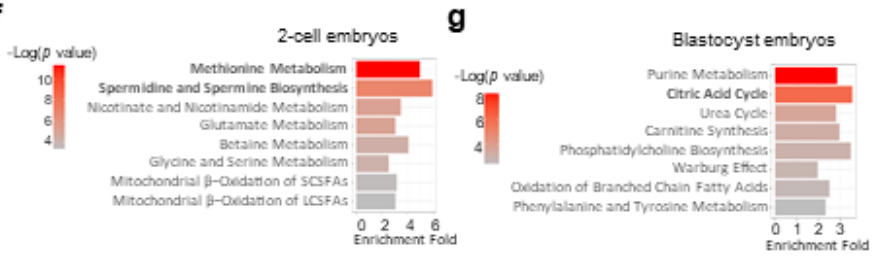

h Overlapped metabolites
in 2-cell embryos \& 2C-like cells igher in 2-cell embryos Higher in 2C-like cel

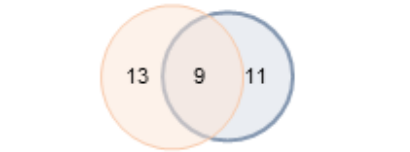

GSH, GSSG, S-Adenosyl-methionine, S-Methyl5 -thioadenosine, Spermidine, N-Acetylputrescine, SDMA-ADMA, UDP-N-acetylglucosamine, 2Deoxycytodine

j

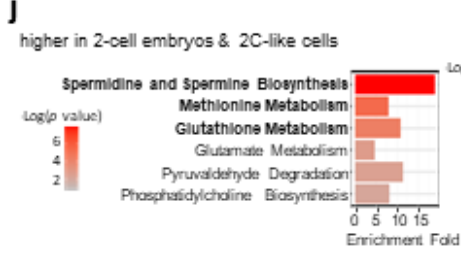

i

Overlapped metabolites in blastocyst embryos \& ES cell higher in blastocyst embryos higher in ES cells

$11 \quad 10 \quad 13$

Xanthosine, Tyrosine, Phenylalanine, malate, Carnitine-C5, Carnitine-C3, Inosine, ValineNorvaline, Isoleucine, Hypoxanthine

k

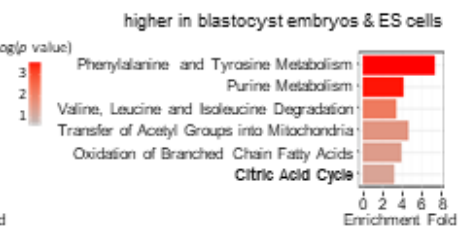

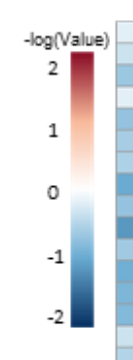
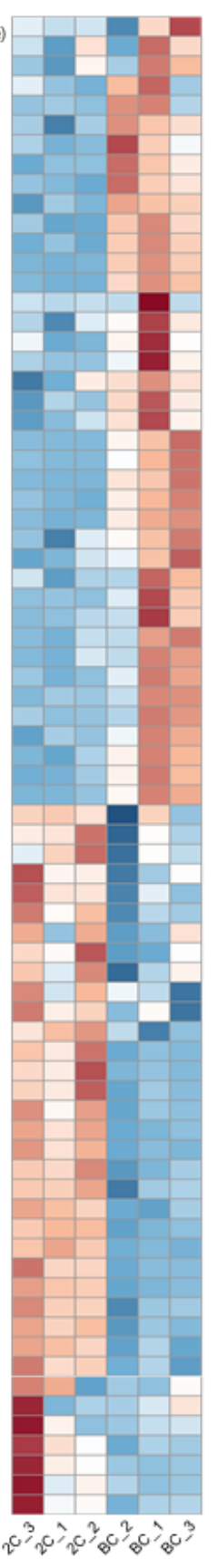

\section{Figure 1}

Figure 1: Metabolomics profiling of 2-cell and blastocyst stage embryos (a) Schematic workflow for sample processing and targeted metabolomics profiling of embryos and ES cells. (b) A principal component analysis (PCA) plot for targeted metabolomics showing clustering of three biological replicates of 2-cell and blastocyst stage embryos, respectively ( $n=96$ metabolites for each sample). (c) Heatmap showing relative abundance of metabolites in 2-cell and blastocyst stage embryos. Peak areas 
for each detected metabolite were normalized against the total ion count of that sample. For each stage, $\mathrm{n}=3$ biological replicates. Each biological replicate was from 100 pooled embryos at either the 2-cell or blastocyst stage. TCA metabolites are shown in bold fonts. Keys: 2C: 2-cell stage embryos. BC: blastocyst stage embryos. (d) Volcano plot showing log2 fold changes (FC) between 2-cell and blastocyst embryos, and p-values for the comparison of each individual metabolite shown in (c). Blue dots (22 metabolites, including $\mathrm{a}-\mathrm{KG}$ ) and red dots (21 metabolites, including SAM and 2-HG) mean significantly increased or decreased metabolites in 2-cell embryos in comparison to blastocysts with a p-value smaller than 0.05 . (e) Relative abundance of 2-HG and a-KG in 2-cell and blastocyst embryos. ( $f, g$ ) Pathway enrichment analysis of metabolites differentially present in 2-cell (f) and blastocyst embryos (g). The analysis was performed using MetaboAnalyst 4.0 81. SCSFAs: Short Chain Saturated Fatty Acids; LCSFAs: Long Chain Saturated Fatty Acids. (h) Venn diagrams showing overlaps of metabolites higher in 2-cell embryos (compared to blastocyst embryos), and higher in 2CLCs sorted from the Dux-induced cell line (compared to ES cells). (i) Venn diagrams showing overlaps of metabolites higher in blastocyst embryos (compared to 2-cell embryos) and higher in ES cells (compared to the 2CLCs sorted from the Dux-induced cell line).

(j,k) Pathway enrichment analysis of the overlapped metabolites in (h) and (i) performed using MetaboAnalyst 4.0 .

a

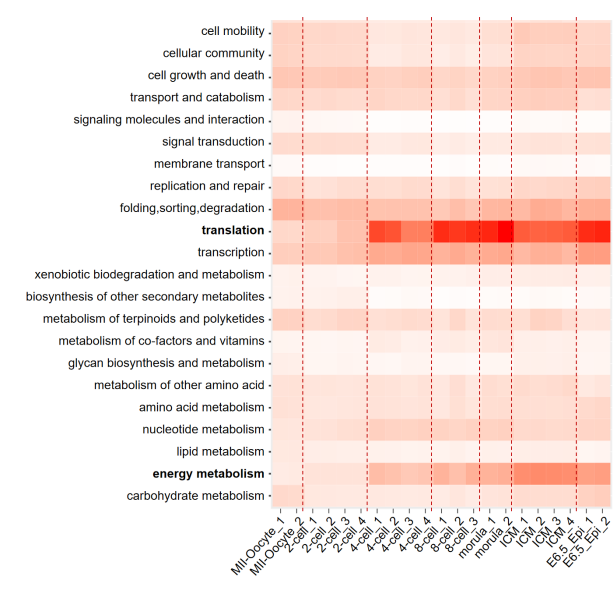

b

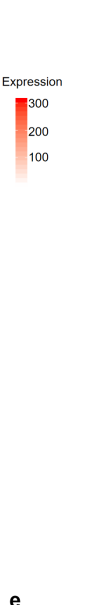

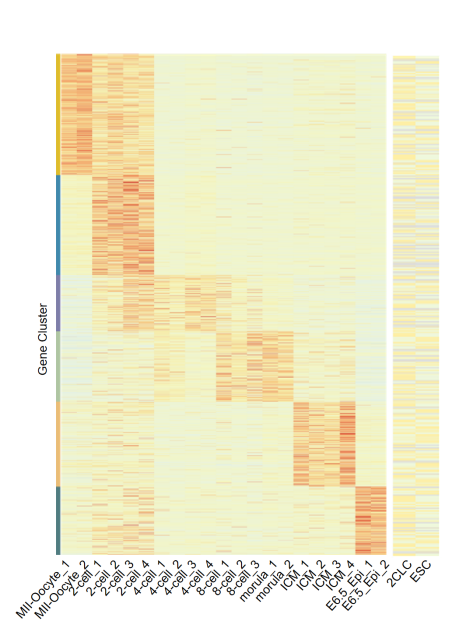
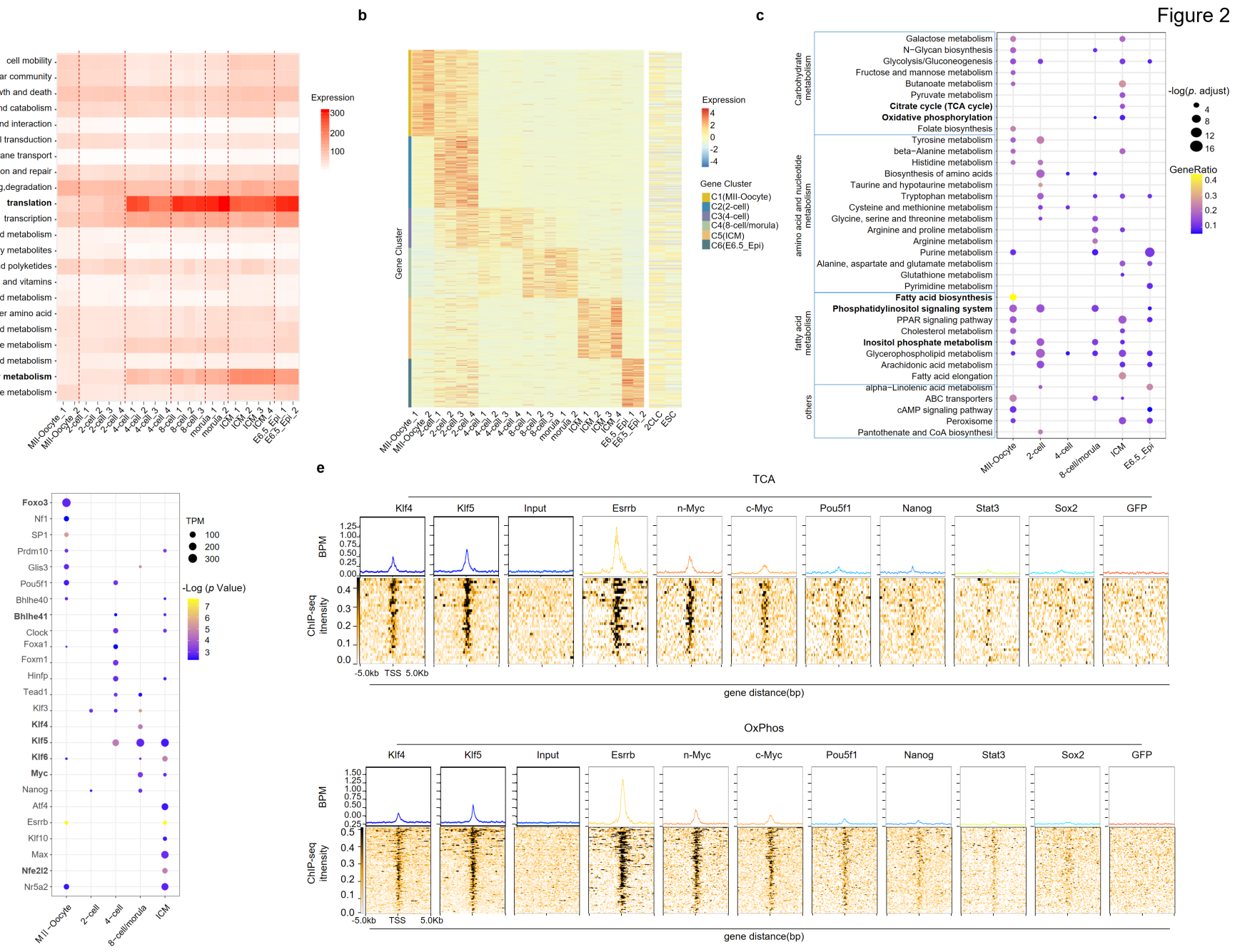

Figure 2 
Figure 2: Genomic and metabolic analysis reveals dynamic metabolic network integrated in the developmental TF network in early embryogenesis (a) Genome-wide KEGG pathway analysis of publicly available bulk RNA-seq with averaged gene expression across indicated pathways showing dynamics for translation and energy metabolism processes across embryonic development stages. MIl: mitotic stage II of oocytes; 2C-8C: 2-cell to 8-cell stage embryos; ICM: Inner cell mass; Epi: Post-implantation epiblast. (b) 3000 metabolism-associated genes were classified into 6 stage-specific clusters by k-means clustering analysis using bulk RNA-seq data as above. The expression of the same genes in the 2-cell like cells (2CLC) and ES cells (ESC) is on the right. (c) KEGG enrichment terms for the 6 stage-specific metabolic gene clusters as shown in (b). (d) TF binding motifs were identified from the stage-specific metabolism gene clusters as illustrated in (b). (e) ChIP-seq analysis showing the read density distribution in a $\pm 5 \mathrm{~kb}$ window around transcription start sites (TSS) of the TCA cycle genes and OxPhos genes bound by key pluripotency transcription factors. GFP is used as a control. BPM: Bins Per Million mapped reads.

Figure 3
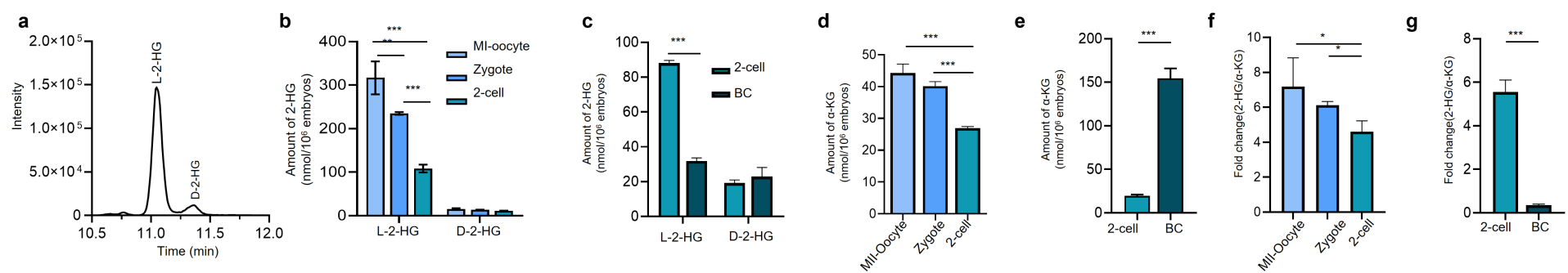

h

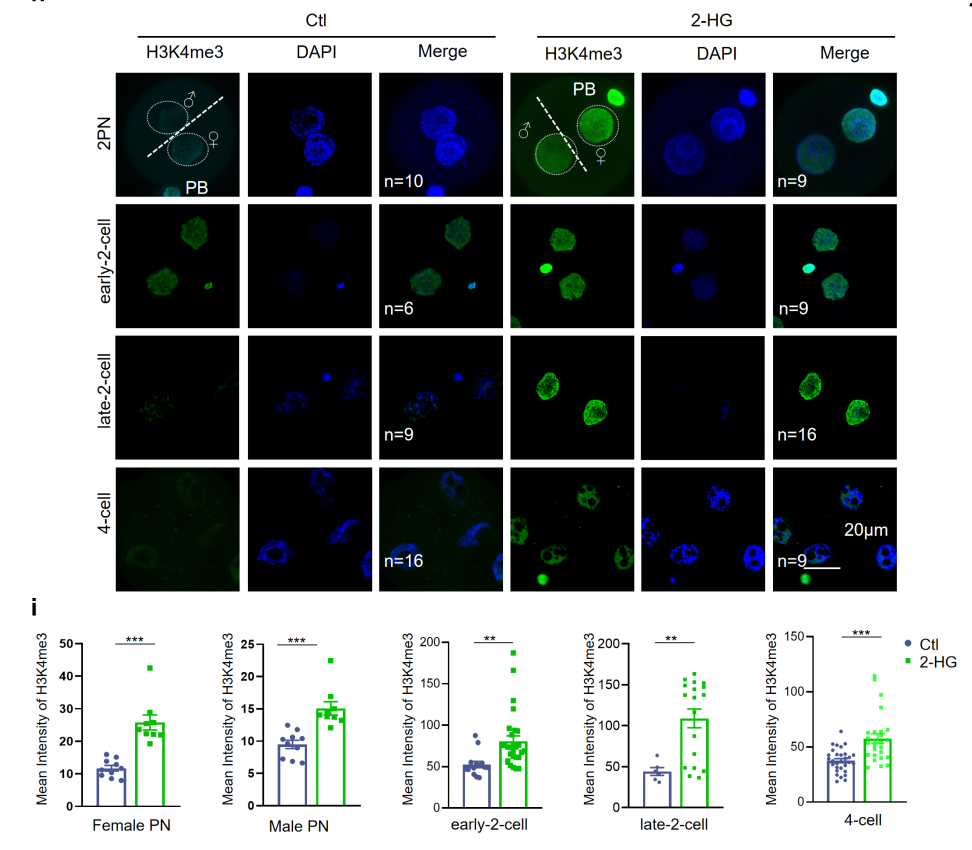

j
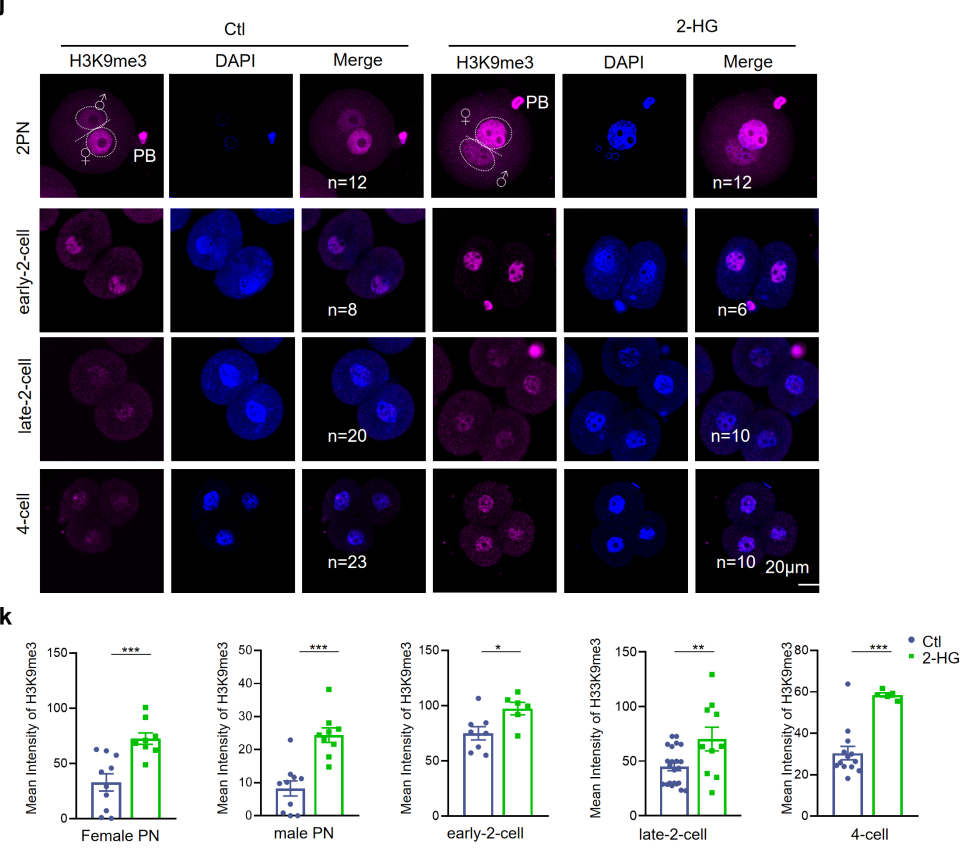

\section{Figure 3}

Figure 3: A decrease of L-2-HG facilitates global erasure of histone methylation during early embryo development. (a) A representative chromatogram of L-2-HG and D-2-HG detected in 2-cell embryos. (b) The absolute level of L-/D- 2-HG in Mll-oocytes, zygote and 2-cell embryos. Data is presented as the mean \pm SEM of three biological replicates. ${ }^{* \star} p<0.002,{ }^{* \star *} p<0.001,(n=3$, unpaired t-test). (c) The absolute level 
of L-2-HG and D-2-HG in 2-cell and blastocyst embryos; Data is presented as the mean \pm SEM of three biological replicates. ${ }^{\star \star \star} p<0.001$, ( $n=3$, unpaired t-test). (d) The absolute level of a-KG in MII-oocytes, zygote and 2-cell embryos. Data is presented as the mean \pm SEM of three biological replicates.

${ }^{* * *} \mathrm{p}<0.001,(\mathrm{n}=3$, unpaired t-test). (e) The absolute level of $\mathrm{a}-\mathrm{KG}$ in 2-cell and blastocyst embryos. Data is presented as the mean \pm SEM of three biological replicates. ${ }^{* *} p<0.001,(n=3$, unpaired t-test). (f) The ratio of 2-HG/a-KG in MIl-oocytes, zygote and 2-cell embryos. Data is presented as the mean $\pm \mathrm{SEM}$, * $\mathrm{p}<0.033$, ( $\mathrm{n}=3$, unpaired t-test). (g) The ratio of 2-HG/a-KG in 2-cell and blastocyst embryos. Data is presented as the mean \pm SEM, ${ }^{* \star *} \mathrm{p}<0.001$, ( $\mathrm{n}=3$, unpaired t-test). (h, i) Control and 0.3 mM L-2-HG-treated zygotes, early 2-cell, early 2-cell and 4-cell embryos stained with an H3K4me3 antibody (green). h:

Representative images of full z-series confocal max projections of embryos from the indicated number of embryos ( $n$ ) in four independent experiments are shown. $\Downarrow$ : maternal pronuclei; $\bowtie$ paternal pronuclei; PB: polar body; 2PN: the 2PN stage of zygote. Scale bar: $20 \mu \mathrm{m}$. i: Quantification of the mean fluorescence intensity for H3K4me3. Data is presented as the mean $\pm \mathrm{SEM},{ }^{\star *} \mathrm{p}<0.002,{ }^{* \star *} \mathrm{p}<0.001$. (j, $\mathrm{k}$ ) Control and 2HG-treated zygotes, early 2-cell, early 2-cell and 4-cell embryos stained with an H3K9me3 antibody (red). j: Representative images of full z-series confocal max projections of embryos from the indicated number of embryos ( $n$ ) in four independent experiments are shown. $\nabla$ : maternal pronuclei; $\bowtie$ paternal pronuclei; PB: polar body; 2PN: the 2PN stage of zygote. Scale bar: $20 \mu \mathrm{m}$. k: Quantification of the mean fluorescence intensity for H3K9me3. Data is presented as the mean $\pm \mathrm{SEM},{ }^{*} \mathrm{p}<0.033,{ }^{* *} \mathrm{p}<0.002,{ }^{* \star *} \mathrm{p}<0.001$.
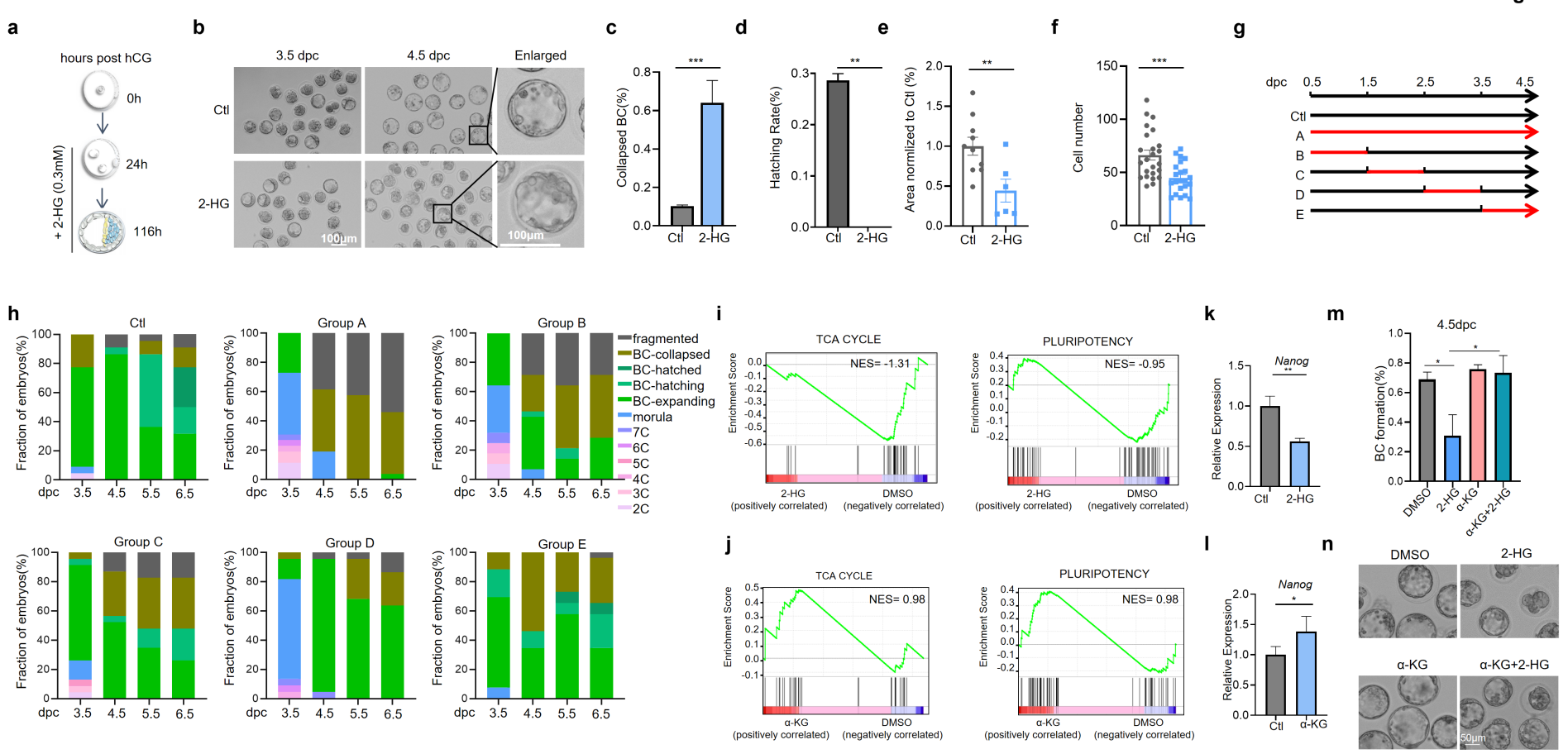

\section{Figure 4}

Figure 4: L-2-HG supplementation impedes early embryo development which is rescuable by a-KG. (a) Schematic illustration of the experimental approach. Zygotes were collected 16 hours after injection of hCG and cultured in KSOM with $0.3 \mathrm{mM} \mathrm{L-2-HG.} \mathrm{The} \mathrm{embryos} \mathrm{were} \mathrm{collected} \mathrm{at} \mathrm{the} \mathrm{indicated} \mathrm{time} \mathrm{for}$ 
further analysis. hCG: human chorionic gonadotropin. (b) Representative DIC (digital image correlation) images of blastocyst stage embryos at $3.5 \mathrm{dpc}$ and $4.5 \mathrm{dpc}$ after the treatment with L-2-HG. This experiment was repeated three times independently. Scale bar: $100 \mu \mathrm{m}$. (c-f) The blastocyst collapsed rate, the hatching rate, the cavity area and the cell number per embryo (counted by DAPI positive cells) in $0.3 \mathrm{mM} \mathrm{L-2-HG-treated} \mathrm{(from} 16$ hours after injection of hCG to the blastocyst stage) and untreated embryos. Error bar represents \pm SEM, ${ }^{\star \star} p<0.002,{ }^{\star \star \star} \mathrm{p}<0.001$. (g) Different schemes of treatment period with $0.3 \mathrm{mM} \mathrm{L-2-HG}$. The $24 \mathrm{hrs}$ time window for L-2-HG treatment is highlighted in red. (h) Stacked bar plots showing fraction of embryos at different developmental stages with the different L-2-HG treatment schemes above. The number of embryos of group A to group $F$ was $22,26,28,23,25,26$. (i, j) Gene set enrichment analysis of the TCA cycle genes and pluripotency genes of L-2-HG-treated (i) or a-KG-treated (j) blastocyst stage embryos in comparison to untreated embryos. NES: Normalized Enrichment Score. (k, I) qRT-PCR showing the pluripotency gene Nanog expression in 2-HG-treated (k) or a-KG-treated (I) blastocyst stage embryos. Five embryos that reach to the blastocyst stage of each condition were pooled for qRT-PCR. Data is presented as the mean \pm SEM of three biological replicates, ${ }^{*} p<0.05$. (m-n) The blastocyst formation rate was determined at $4.5 \mathrm{dpc}$ for embryos treated with $0.3 \mathrm{mM} \mathrm{L-2-HG}$ alone, 0.15 $\mathrm{mM}$ a-KG alone, or a-KG on top of L-2-HG from 24 hours after $\mathrm{hCG}$ injection to $4.5 \mathrm{dpc}$. 
a

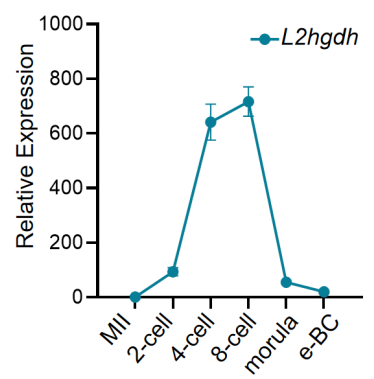

e

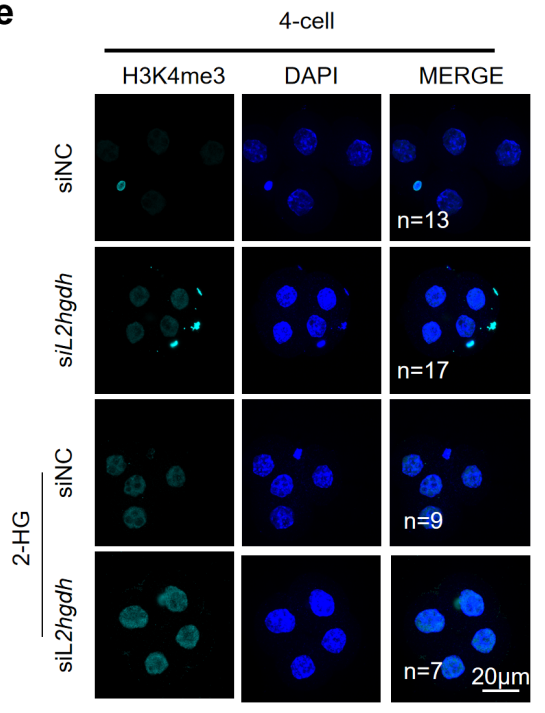

C

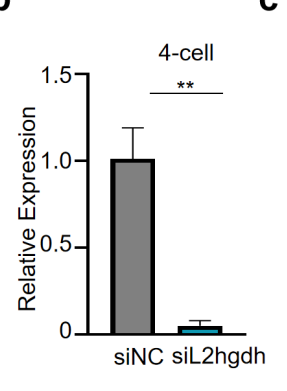

Figure 5

d

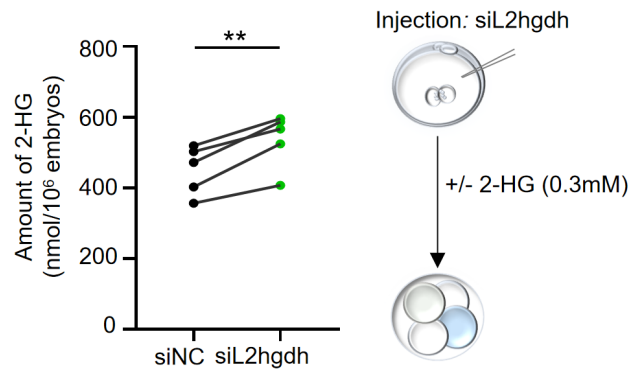

\section{$\mathbf{f}$}

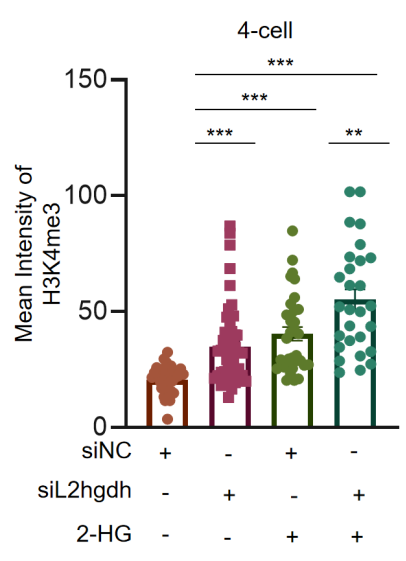

\section{Figure 5}

Figure 5: L2hgdh deficiency impedes H3K4me3 methylation erasure during mouse early embryo development (a) qRT-PCR with RNA from embryos showing the L2hgdh mRNA level at different developmental stages. Five embryos were collected and pooled for one biological replicate. Data is presented as the mean \pm SEM of three biological replicates from a representative experiment. (b) qRT-PCR showing the L2hgdh mRNA level in 4-cell embryos after zygotic injection of siRNAs. Scrambled siRNA 
duplex without specific targets was used as a negative control (siNC). Five embryos were collected and pooled for one biological replicate. Data is presented as the mean $\pm S E M$ of three biological replicates from a representative experiment, ${ }^{* *} \mathrm{p}<0.01$ by t-test. (c) L-2-HG level determined by MS in 4-cell embryos after zygotic injection of siRNAs. (d) Schematic diagram of L2hgdh siRNA injection at zygotes and cultured in KSOM supplemented with L-2-HG. Immunofluorescence and qRT-PCR at 4-cell embryos were performed. (e, f) Immunostaining of H3K4me3 (Cyan) in siNC, siL2hgdh, siNC + L-2-HG and siL2hgdh + L2-HG in 4-cell embryos. e: Representative full z-series confocal projection images of embryos from the indicated number of embryos ( $\mathrm{n}$ ) in two independent experiments are shown. Scale bar $20 \mu \mathrm{m}$. $\mathrm{f}$ :

Quantification of the mean fluorescence intensity for $\mathrm{H} 3 \mathrm{~K} 4 \mathrm{me} 3$ is shown besides the images. ${ }^{*} \mathrm{p}<0.033$, ${ }^{\star \star} p<0.002,{ }^{\star \star *} \mathrm{p}<0.001$ by $\mathrm{t}$-test.

\section{Supplementary Files}

This is a list of supplementary files associated with this preprint. Click to download.

- ExtendedDataFig.1.tif

- ExtendedDataFig.2.tif

- ExtendedDataFig.3.tif

- ExtendedDataFig.4.tif

- ExtendedDataFig.5del.tif

- ExtendedDataFig.6.tif

- ExtendedDataFig.7.tif

- ExtendedDataFig.8.tif 